Revista de Matemática: Teoría y Aplicaciones 2017 24(1) : 29-44

CIMPA - UCR ISSN: 1409-2433 (PRINT), 2215-3373 (ONLINE)

\title{
LA DINÁMICA LINEAL DE UNA N-UPLA DE OPERADORES HIPERCÍCLICA COMO GENERADORA DE LA BIHIPERCICLIDAD
}

\author{
THE LINEAR DYNAMIC OF A HYPERCYCLIC \\ TUPLE OF OPERATORS SUCH AS \\ BIHYPERCYCLICITY GENERATOR
}

\author{
NELYDA VARGAS-DUQUE*
}

Received: 12/Feb/2015; Revised: 16/Mar/2016;

Accepted: 13/Apr/2016

\begin{abstract}
Revista de Matemática: Teoría y Aplicaciones is licensed under a Creative Commons Reconocimiento-NoComercial-Compartirigual 4.0 International License.

Creado a partir de la obra en http://www.revistas.ucr.ac.cr/index.php/matematica

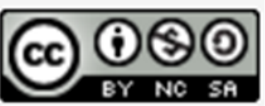

*Universidad de los Andes, Mérida, Venezuela. E-Mail: nelidav@ula.ve, nelida2785@gmail.com
\end{abstract}




\begin{abstract}
Resumen
En este artículo se estudia el estado del arte del trabajo reciente de Grosse-Erdmann \& Kim [6], destacando como una n-upla de operadores hipercíclica es una fuente para construir funciones bilineales bihipercíclicas.
\end{abstract}

Palabras clave: órbita densa; n-upla de operadores hipercíclica; función bilineal bihipercíclica.

\begin{abstract}
In this paper we study the art of recent work Grosse-Erdmann \& Kim [6], highlighting as a hypercyclic tuples of operators is a source to build bihypercyclic bilinear mappings.
\end{abstract}

Keywords: dense orbit; hypercyclic tuples of operators; bihypercyclic bilinear mapping.

Mathematics Subject Classification: 37C35, 46E20 , 47D03.

\title{
1 Introducción
}

Este trabajo es un tema de reciente investigación, en el cual se analiza la noción de órbita para funciones bilineales y se prueban algunos resultados de la bihiperciclicidad que son análogos con la hiperciclicidad, estos son: la bihiperciclicidad se preserva bajo la conjugación, el conjunto de los vectores bihipercíclicos no es denso, el Teorema de León y Muller es aplicable en la bihiperciclicidad con ciertas condiciones y se cumple la propiedad de cuasiconjugada. Asimismo, se desarollan varios ejemplos de estas funciones en los espacios $\ell^{1}$ y $\ell^{2}$; destacando que una $n$-upla hipercíclica, constituye una de las fuentes para construir funciones bilineales bihipercíclicas. La teoría que fundamenta los estudios de hiperciclicidad, se pueden consultar en [2], [1], [10], [5], [12], [11] y [7]. En cuanto a la bihiperciclicidad ver [6].

\section{Teoría y resultados}

A lo largo del artículo, un espacio de Hilbert separable se denotará como $X$, el conjunto de operadores lineales acotados sobre $X$ se denotará como $\mathcal{L}(X)$ y se asume que las funciones bilineales son continuas. 
Definición 1. Una función $B: X \times X \rightarrow X$ es bilineal, si es lineal en la primera variable y lineal en la segunda variable, es decir, si para todo $u, v, w$ en $X$ y escalares $\alpha$ y $\beta$, se cumple

$$
\begin{aligned}
& B(\alpha u+\beta v, w)=\alpha B(u, w)+\beta B(v, w) \\
& B(u, \alpha v+\beta w)=\alpha B(u, v)+\beta B(u, w) .
\end{aligned}
$$

Asimismo, una función bilineal es simétrica si $B(u, v)=B(v, u)$.

Definición 2. Sea $B: X \times X \rightarrow X$ una función bilineal, $B$ es acotada si existe una constante $C>0$, tal que, para todo $x, y \in X$,

$$
\|B(x, y)\| \leq C\|x\|\|y\| .
$$

Es importante mencionar, que si $B$ es acotada es equivalente a decir que $B$ es continua, como sucede en el caso lineal. Del mismo modo, se cumple, que las funciones bilineales en espacios de dimensión finita son continuas. Para abordar el comportamiento dinámico de las funciones bilineales, se estudian sus órbitas.

Definición 3. Sea $B: X \times X \rightarrow X$ una función bilineal, dado un par $(x, y) \in$ $X \times X$, la órbita de $(x, y)$ bajo $B$ es el conjunto

$$
\operatorname{orb}((x, y), B)=\bigcup_{n=0}^{\infty} U_{n},
$$

donde

$$
\begin{aligned}
& U_{0}=\{x, y\} \\
& U_{1}=\{x, y, B(x, x), B(x, y), B(y, x), B(y, y)\} \\
& U_{2}=\{x, y, B(x, x), B(x, y), B(y, x), B(y, y), B(x, B(x, x)) \\
& B(x, B(x, y)), \ldots, B(y, B(x, x)), B(y, B(x, y)), \ldots, B(B(x, x), x), \\
&B(B(x, x), y), B(B(x, x), B(x, x)), \ldots\}, \\
& y \text { en general para } n \geq 1, U_{n}=U_{n-1} \bigcup\left\{B(u, v): u, v \in U_{n-1}\right\} .
\end{aligned}
$$

Otra forma alternativa de definir la órbita para funciones bilineales es la siguiente, dado un $(x, y) \in X \times X$, la órbita de $(x, y)$ bajo $B$ es el conjunto

$$
\operatorname{orb}((x, y), B)=\bigcup_{n=0}^{\infty}[B]_{n}(x, y)
$$


donde

$$
\begin{aligned}
{[B]_{0}(x, y)=} & \{x, y\} \\
{[B]_{1}(x, y)=} & \{B(x, x), B(x, y), B(y, x), B(y, y)\} \\
{[B]_{2}(x, y)=} & \{B(x, B(x, x)), B(x, B(y, y)), B(y, B(y, x)), \ldots, \\
& B(B(x, y), x), B(B(x, y), y), B(B(y, y), x), \ldots\}
\end{aligned}
$$

y en general para $n \geq 1,[B]_{n}(x, y)=\{B(u, v): \exists j \in\{0, \ldots, n-1\}$ tal que los elementos de $\left.u \in[B]_{j}(x, y), v \in[B]_{n-1-j}(x, y)\right\}$, por lo tanto, $[B]_{n}(x, y)$ contiene todos los elementos de la órbita que son obtenidos exactamente por $n$ aplicaciones. Esta definición de órbita es una propuesta introducida por primera vez en [6], los autores destacan que es una alternativa natural pero posiblemente no única. Dicha representación de órbita es la que será utilizada para las pruebas, ya que es más práctica en el uso del método de inducción.

Definición 4. Sea $B: X \times X \rightarrow X$ una función bilineal, se llama bihipercíclica si existe un par $(x, y) \in X \times X$, tal que orb $((x, y), B)$ es densa en $X$.

A tal par $(x, y)$ se le denomina vector bihipercíclico para $B$ y al conjunto de esos vectores bihipercíclicos se denotará como $B H C(B)$.

Un resultado en la hiperciclicidad es que el conjunto de los vectores hipercíclicos es siempre un conjunto $G_{\delta}$ denso cuando este no es vacío, ver [2]. Lo análogo no es cierto para bihiperciclicidad, ya que el conjunto de vectores bihipercíclicos es un conjunto $G_{\delta}$ pero este no es denso.

Teorema 1. El conjunto $B H C(B)$ es un conjunto $G_{\delta}$ en $X \times X$ que no es denso. Más precisamente, si $M>0$ tal que para toda $x, y \in X,\|B(x, y)\| \leq$ $M\|x\|\|y\| y\|x\|,\|y\| \leq \frac{1}{M}$ entonces $(x, y)$ no es un par bihipercíclico para $B$.

Demostración. Primero se demostrará que $B H C(B)$ se puede escribir como la intersección de una colección numerable de abiertos, es decir, que es un conjunto $G_{\delta}$. Como $X$ es separable existe una base numerable de abiertos $\left(V_{k}\right)_{k \geq 1}$ para la topología de $X$. Sea $\left(x^{\prime}, y^{\prime}\right) \in X \times X$ un par bihipercíclico, es decir,

$$
\begin{aligned}
\left(x^{\prime}, y^{\prime}\right) \in B H C(B) & \Leftrightarrow \forall k \in \mathbb{N}: \operatorname{orb}\left(\left(x^{\prime}, y^{\prime}\right), B\right) \cap V_{k} \neq \emptyset, \\
& \Leftrightarrow \forall k \in \mathbb{N}:\left(\bigcup_{n=0}^{\infty}[B]_{n}\left(x^{\prime}, y^{\prime}\right)\right) \cap V_{k} \neq \emptyset, \\
& \Leftrightarrow \forall k \in \mathbb{N}, \exists n \in \mathbb{N}_{0}:[B]_{n}\left(x^{\prime}, y^{\prime}\right) \cap V_{k} \neq \emptyset, \\
& \Leftrightarrow\left(x^{\prime}, y^{\prime}\right) \in \bigcap_{k=1}^{\infty} \bigcup_{n=0}^{\infty}\left\{(x, y):[B]_{n}(x, y) \cap V_{k} \neq \emptyset\right\} .
\end{aligned}
$$


Luego por la continuidad de $B$, se tiene que $B H C(B)$ es la intersección de una colección numerable de abiertos, es decir,

$$
B H C(B)=\bigcap_{k=1}^{\infty} \bigcup_{n=0}^{\infty}\left\{(x, y):[B]_{n}(x, y) \cap V_{k} \neq \emptyset\right\}
$$

y por lo tanto es un conjunto $G_{\delta}$.

Ahora se demostrará que $B H C(B)$ no es denso. Sea $(x, y) \in X \times X$ con $U$ definido como $\left\{B(x, y):\|x\|,\|y\| \leq \frac{1}{M}\right\}$, se demostrará que $U$ no contiene ningún vector bihipercíclico, es decir,

$$
\text { para cualquier } z \in \operatorname{orb}((x, y), B)=\bigcup_{n=0}^{\infty}[B]_{n}(x, y) \text { se cumple }\|z\| \leq \frac{1}{M} \text {, }
$$

lo cual implica que $(x, y)$ no es bihipercíclico para $B$ y por lo tanto $B H C(B)$ no es denso. Para ver esto, se procede por inducción.

Para $n=0$, se tiene que

$$
z \in[B]_{0}(x, y)=\{x, y\},\|z\| \leq \frac{1}{M} .
$$

Suponga que (1) se cumple para toda $k<n$, así, si $z \in[B]_{k}(x, y)$ se tiene que $\|z\| \leq \frac{1}{M}$.

Luego para $k=n$, sea $z \in[B]_{n}(x, y)$, entonces hay $j \in\{0, \ldots, n-1\}$, tal que $u \in[B]_{j}(x, y), v \in[B]_{n-1-j}(x, y)$, así $z=B(u, v), \mathrm{y}$

$$
\|z\|=\|B(u, v)\| \leq M\|u\|\|v\| \leq \frac{1}{M}
$$

por lo tanto, para cualquier $z \in \operatorname{orb}((x, y), B)$, se cumple que $\|z\| \leq \frac{1}{M}$, así $(x, y)$ no es un vector bihipercíclico para $B$.

Otro resultado en la hiperciclicidad es el de León \& Muller [9], el cual dice lo siguiente: si $T$ es un operador hipercíclico entonces $\lambda T$ también es hipercíclico con $\lambda \in \mathbb{C},|\lambda|=1$. Por otra parte, si $|\lambda| \neq 1$, en general, $\lambda T$ no es hipercíclico. Para bihiperciclicidad tiene un desarrollo distinto, como se expone en el siguiente Teorema.

Teorema 2. Sea $B$ una función bilineal continua bihipercílcica y $\lambda \neq 0$ un escalar. Entonces $\lambda B$ es también bihipercíclica. Por otra parte,

$$
B H C(\lambda B)=\left\{\left(\frac{x}{\lambda}, \frac{y}{\lambda}\right):(x, y) \in B H C(B)\right\} .
$$


Demostración. Se demostrará que $\lambda B$ es bihipercíclica con $\lambda \neq 0$, de tal manera, debe existir un $\left(x^{\prime}, y^{\prime}\right) \in X \times X$ tal que

$$
\operatorname{orb}\left(\left(x^{\prime}, y^{\prime}\right), \lambda B\right)
$$

es densa en $X$.

Sea $\left(x^{\prime}, y^{\prime}\right)=\left(\frac{x}{\lambda}, \frac{y}{\lambda}\right) \operatorname{con}(x, y) \in B H C(B)$, entonces se demostrará que

$$
\operatorname{orb}\left(\left(\frac{x}{\lambda}, \frac{y}{\lambda}\right), \lambda B\right)
$$

es densa en $X$.

Primero, se demuestra que para cualquier $n \geq 0, x, y \in X$ y $\lambda \neq 0$,

$$
[\lambda B]_{n}\left(\frac{x}{\lambda}, \frac{y}{\lambda}\right)=\frac{1}{\lambda}[B]_{n}(x, y) .
$$

Se procede por inducción, para $n=0$, se tiene

$$
[\lambda B]_{0}\left(\frac{x}{\lambda}, \frac{y}{\lambda}\right)=\left\{\frac{x}{\lambda}, \frac{y}{\lambda}\right\}=\frac{1}{\lambda}\{x, y\}=\frac{1}{\lambda}[B]_{0}(x, y) .
$$

Suponga que (2) se cumple para toda $k<n$. Entonces para $k=n$,

$$
\begin{aligned}
& {[\lambda B]_{n}\left(\frac{x}{\lambda}, \frac{y}{\lambda}\right)=} \\
& =\left\{\lambda B(u, v): \exists j \in\{0, \ldots, n-1\}, u \in[\lambda B]_{j}\left(\frac{x}{\lambda}, \frac{y}{\lambda}\right), v \in[\lambda B]_{n-1-j}\left(\frac{x}{\lambda}, \frac{y}{\lambda}\right)\right\} \\
& =\left\{\lambda B(u, v): \exists j \in\{0, \ldots, n-1\}, u \in \frac{1}{\lambda}[B]_{j}(x, y), v \in \frac{1}{\lambda}[B]_{n-1-j}(x, y)\right\} \\
& =\left\{\lambda B\left(\frac{u^{\prime}}{\lambda}, \frac{v^{\prime}}{\lambda}\right): \exists j \in\{0, \ldots, n-1\}, u^{\prime} \in[B]_{j}(x, y), v^{\prime} \in[B]_{n-1-j}(x, y)\right\} \\
& =\frac{1}{\lambda}\left\{B\left(u^{\prime}, v^{\prime}\right): \exists j \in\{0, \ldots, n-1\}, u^{\prime} \in[B]_{j}(x, y), v^{\prime} \in[B]_{n-1-j}(x, y)\right\} \\
& =\frac{1}{\lambda}[B]_{n}(x, y) .
\end{aligned}
$$

Por lo tanto, para toda $n \geq 0$

$$
[\lambda B]_{n}\left(\frac{x}{\lambda}, \frac{y}{\lambda}\right)=\frac{1}{\lambda}[B]_{n}(x, y),
$$

de tal manera que

$$
\bigcup_{n=0}^{\infty}[\lambda B]_{n}\left(\frac{x}{\lambda}, \frac{y}{\lambda}\right)=\frac{1}{\lambda} \bigcup_{n=0}^{\infty}[B]_{n}(x, y)
$$


Ahora como $B$ es bihipercíclica, existe un $(x, y) \in X \times X$ tal que $\operatorname{orb}((x, y), B)$ es densa en $X$, luego que, $\frac{1}{\lambda} \operatorname{orb}((x, y), B)=\frac{1}{\lambda} \bigcup_{n=0}^{\infty}[B]_{n}(x, y)$ es densa en $X$, en efecto $\bigcup_{n=0}^{\infty}[\lambda B]_{n}\left(\frac{x}{\lambda}, \frac{y}{\lambda}\right)$ también es densa en $X$ y por lo tanto $\lambda B$ es bihipercíclica.

A continuación se introduce la definición de cuasiconjugada y conjugada para funciones bilineales.

Definición 5. [6] Sea $C: X \times X \rightarrow X$ y $B: Y \times Y \rightarrow Y$ funciones bilineales.

a) Entonces $C$ es cuasiconjugada a $B$ si existe una aplicación continua definida como $\phi: Y \rightarrow X$ con rango denso tal que para toda $x, y \in Y$, se cumple que $C(\phi(x), \phi(y))=\phi(B(x, y))$, esto es, el siguiente diagrama conmuta.

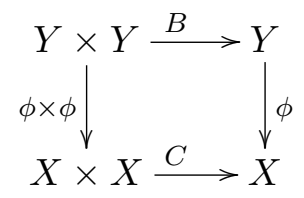

b) Si $\phi$ es un homeomorfismo entonces $B$ y $C$ son llamadas conjugadas.

La noción de transformación cuasiconjugada es una herramienta muy útil, ya que si un operador lineal tiene una propiedad y es cuasiconjugado a otro operador lineal, entonces este hereda la propiedad. En particular, la hiperciclicidad se preserva bajo la cuasiconjugación (envía vectores hipercíclicos a vectores hipercíclicos, ver [2]), lo análogo es aplicable en funciones bilineales.

Teorema 3. Sea $B$ una función bilineal bihipercíclica y $C$ una función bilineal que es cuasiconjugada a B. Entonces $C$ también es bihipercíclica. Más precisamente, si $(x, y)$ es bihipercíclico para $B$ entonces $(\phi(x), \phi(y))$ es bihipercíclico para $C$.

Demostración. Primero se demostrará, que para cualquier $x, y \in Y$,

$$
\operatorname{orb}((\phi(x), \phi(y)), C)=\phi(\operatorname{orb}((x, y), B)) .
$$

Sólo basta demostrar, que para cualquier $x, y \in Y$, y $n \geq 0$

$$
[C]_{n}(\phi(x), \phi(y))=\phi\left([B]_{n}(x, y)\right)
$$

Se procede por inducción. Para $n=0$, se tiene

$$
[C]_{0}(\phi(x), \phi(y))=\{\phi(x), \phi(y)\}=\phi(\{x, y\})=\phi\left([B]_{0}(x, y)\right)
$$


Ahora, sea $n \geq 1$. Suponga que (3) se cumple para toda $k<n$. Entonces para $k=n$,

$$
\begin{aligned}
& {[C]_{n}(\phi(x), \phi(y))=} \\
&=\{C(u, v): \exists j \in\{0, \ldots, n-1\}, \\
&\left.u \in[C]_{j}(\phi(x), \phi(y)), v \in[C]_{n-1-j}(\phi(x), \phi(y))\right\} \\
&=\left\{C(u, v): \exists j \in\{0, \ldots, n-1\}, u \in \phi\left([B]_{j}(x, y)\right), v \in \phi\left([B]_{n-1-j}(x, y)\right)\right\} \\
&=\left\{C\left(\phi\left(u^{\prime}\right), \phi\left(v^{\prime}\right)\right): \exists j \in\{0, \ldots, n-1\}, u^{\prime} \in[B]_{j}(x, y), v^{\prime} \in[B]_{n-1-j}(x, y)\right\} \\
&= \phi\left(\left\{B\left(u^{\prime}, v^{\prime}\right): \exists j \in\{0, \ldots, n-1\}, u^{\prime} \in[B]_{j}(x, y), v^{\prime} \in[B]_{n-1-j}(x, y)\right\}\right) \\
&= \phi\left([B]_{n}(x, y)\right) .
\end{aligned}
$$

Por lo tanto, para toda $n \geq 0$,

$$
[C]_{n}(\phi(x), \phi(y))=\phi\left([B]_{n}(x, y)\right)
$$

así

$$
\bigcup_{n=0}^{\infty}[C]_{n}(\phi(x), \phi(y))=\phi\left(\bigcup_{n=0}^{\infty}[B]_{n}(x, y)\right),
$$

de tal manera que

$$
\operatorname{orb}((\phi(x), \phi(y)), C)=\phi(\operatorname{orb}((x, y), B)) .
$$

Ahora se demostrará que si $B$ es bihipercíclica, $B$ y $C$ son cuasiconjugadas entonces $C$ es bihipercíclica. Como $B$ es bihipercíclica, entonces existe un par o un vector $(x, y) \in Y \times Y$ tal que $\operatorname{orb}((x, y), B)$ es densa en $Y$, así se tiene que $\phi(\overline{\operatorname{orb}((x, y), B)})=\phi(Y)$ y $\phi$ con rango denso se tiene $\overline{\phi(Y)}=X$.

Luego,

$$
\begin{aligned}
X & =\overline{\phi(Y)}=\overline{\phi(\overline{\operatorname{orb}((x, y), B)})} \subset \phi(\overline{\operatorname{orb}((x, y), B)})=\overline{\operatorname{orb}((\phi(x), \phi(y)), C)} \\
& \subset X
\end{aligned}
$$

Por lo tanto, $\overline{\operatorname{orb}((\phi(x), \phi(y)), C)}=X$, así $C$ es bihipercíclica y se tiene que $(\phi(x), \phi(y)) \in B H C(C)$.

Ejemplo 1. Para cualquier $\lambda \neq 0, B$ y $\lambda B$ son funciones bilineales conjugadas via $\phi(x)=\frac{1}{\lambda} x$.

Primero se demostrará que $B$ es cuasiconjugada a $\lambda B$, con $\lambda \neq 0$. 
Sea $\phi: Y \rightarrow X$ definida como $\phi(x)=\frac{1}{\lambda} x$, una aplicación continua con rango denso. Para toda $x, y \in Y$, se cumple que

$$
\begin{aligned}
\lambda B(\phi(x), \phi(y)) & =\lambda B\left(\frac{1}{\lambda} x, \frac{1}{\lambda} y\right) \\
& =\frac{1}{\lambda} B(x, y) \\
\phi(B(x, y)) & =\frac{1}{\lambda} B(x, y)
\end{aligned}
$$

y por lo tanto $\lambda B(\phi(x), \phi(y))=\phi(B(x, y))$, esto es, el siguiente diagrama conmuta.

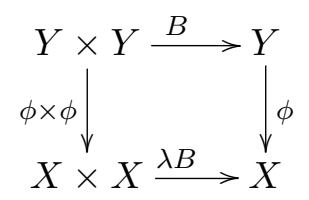

En efecto $B$ es cuasiconjugada a $\lambda B$, con $\lambda \neq 0$. Adicionalmente, como $\phi(x)$ es biyectiva y $\phi^{-1}(x)$ es continua, se tiene que $\phi(x)$ es un homeomorfismo, lo cual implica que $B$ es conjugada a $\lambda B$, con $\lambda \neq 0$, via $\phi(x)=\frac{1}{\lambda} x$.

\section{Ejemplos de funciones bilineales bihipercíclicas}

En esta sección se mostrarán los primeros ejemplos de funciones bihipercíclicas.

Proposición 1. Sea $B$ una función bilineal tal que hay algún $x \in X$ para el cual el operador $T=B(x, \cdot): X \rightarrow X$ es hipercíclico. Entonces $B$ es bihipercíclica. Más precisamente, si y es un vector hipercíclico para $T$ entonces $(x, y)$ es bihipercíclico para $B$.

Demostración. Sea $y \in H C(T)$, lo cual implica que la orb $(y, T)$ es densa en $X$. Los elementos de la órbita de $y \in X$ bajo $T$ son de la forma: $T y=B(x, y)$, $T^{2} y=B(x, B(x, y)), T^{3} y=B(x, B(x, B(x, y)))$, .., así se puede deducir que $\operatorname{orb}(y, T) \subset \operatorname{orb}((x, y), B)$ y por lo tanto, $(x, y)$ es un par bihipercíclico para $B,(x, y) \in B H C(B)$.

A continuación, se presenta el estudio de las funciones bilineales continuas sobre $\ell^{2} \times \ell^{2}$ que pueden ser bihipercíclicas, usando la siguiente definición, $f \otimes g(v, w)=f(v) g(w)$, donde $f$ es un funcional y $g$ un operador sobre $X$.

Ejemplo 2. Sea $T$ un operador hipercíclico sobre $X$ y $x^{*} \neq 0$ un funcional lineal continuo sobre $X$. Entonces la función bilineal

$$
B=x^{*} \otimes T
$$

es bihipercíclica. 
En forma general, se tiene que $B(x, y)=x^{*} \otimes T(x, y)=x^{*}(x) T(y)$. En efecto, sea $x \in X$ tal que $x^{*}(x)=1$, así $B(x, y)=T(y)$ y por Proposición 1 con $y$ un vector hipercíclico para $T$ entonces $(x, y)$ es un vector bihipercíclico para $B$. Por lo tanto, $B$ es bihipercíclica.

En particular, sea $\mathcal{B}: \ell^{2} \rightarrow \ell^{2}$ el operador traslación a la izquierda, se define $T=2 \mathcal{B}, T$ es hipercíclico. Este resultado fue desarrollado por Rolewicz en 1969, y es el primer operador lineal hipercíclico en espacios de Banach. Ahora, sea $x^{*}: \ell^{2} \rightarrow \mathbb{C}$ un funcional lineal continuo, el cual puede ser representado en términos del producto interior, donde para cualquier $x \in \ell^{2}$, existe un $y^{\prime} \in \ell^{2}$, tal que

$$
x^{*}(x)=<x, y^{\prime}>\text {; }
$$

en efecto, tomando $x=\left(x_{1}, x_{2}, x_{3}, \ldots\right) \in \ell^{2}, y^{\prime}=(1,0,0,0, \ldots) \in \ell^{2}$, se tiene que $x^{*}(x)=x_{1}$.

Luego, con $y=\left(y_{1}, y_{2}, y_{3}, \ldots\right) \in \ell^{2}$, se halla (1), usando la definición de $\otimes, x^{*}$ y $T$, donde se tiene

$$
\begin{aligned}
B(x, y) & =x^{*} \otimes T(x, y) \\
& =x^{*}(x) T(y) \\
& =x_{1}\left(2 y_{2}, 2 y_{3}, \ldots\right) \\
& =2 x_{1} \mathcal{B}(y)
\end{aligned}
$$

por lo tanto $B$ es bihipercíclica, ya que $\operatorname{orb}(y, T) \subset \operatorname{orb}((x, y), B)$. De la misma manera, si $x_{1}=1, B(x, y)=2 \mathcal{B}(y)$, con $y$ un vector hipercíclico para $2 \mathcal{B}$ entonces $(x, y)$ es un vector bihipercíclico para $B$.

Ejemplo 3. Sea $T$ un operador hipercíclico no inyectivo sobre $X, x \in X$, con $x \neq 0$, tal que $T x=0$ y sea $x^{*} \neq 0$ un funcional lineal continuo sobre $X$ con $x^{*}(x)=1$. Entonces

$$
B=x^{*} \otimes T+T \otimes x^{*}
$$

es bihipercíclica y simétrica.

En forma general, sea $x \in X, x \neq 0$ tal que $T x=0$ y $x^{*}(x)=1$, así se tiene que

$$
\begin{aligned}
B(x, y) & =\left(x^{*} \otimes T+T \otimes x^{*}\right)(x, y) \\
& =x^{*} \otimes T(x, y)+T \otimes x^{*}(x, y) \\
& =x^{*}(x) T(y)+T(x) x^{*}(y) \\
& =1 T(y)+0 x^{*}(y) \\
& =T(y) .
\end{aligned}
$$


y por Proposición 1 con $y$ un vector hipercíclico para $T$ entonces $(x, y)$ es un vector bihipercíclico para $B$. Por lo tanto, $B$ es bihipercíclica. Además, se tiene que $B(x, y)=B(y, x)$ y por lo tanto $B$ es simétrica.

En particular, sea $\mathcal{B}: \ell^{2} \rightarrow \ell^{2}$ el operador traslación a la izquierda, $T=2 \mathcal{B}$ es hipercíclico. Hallando (4) se toma del Ejemplo anterior las definiciones de $\otimes, x^{*}, x$ y $y$, se tiene,

$$
\begin{aligned}
B(x, y) & =\left(x^{*} \otimes T+T \otimes x^{*}\right)(x, y), \\
& =x^{*} \otimes T(x, y)+T \otimes x^{*}(x, y), \\
& =x^{*}(x) T(y)+T(x) x^{*}(y), \\
& =x_{1}\left(2 y_{2}, 2 y_{3}, \ldots\right)+\left(2 x_{2}, 2 x_{3}, \ldots\right) y_{1}, \\
& =2 x_{1} \mathcal{B}(y)+2 y_{1} \mathcal{B}(x), \\
& =2 x_{1} \mathcal{B}(y)+2 y_{1} \mathcal{B}(x),
\end{aligned}
$$

así $B$ es bihipercíclica, ya que $\operatorname{orb}(y, T) \subset \operatorname{orb}((x, y), B)$. De la misma manera, por hipótesis se tiene que $x^{*}(x)=1$ y $T x=0$, así $B(x, y)=T y=2 \mathcal{B}(y)$, siendo $y$ un vector hipercíclico para $T$ entonces $(x, y)$ es un vector bihipercíclico para $B$. Además, como $B(x, y)=B(y, x)$ entonces $B$ es simétrica.

Ahora se estudian funciones bilineales continuas sobre $\ell^{1} \times \ell^{1}$ que pueden ser bihipercíclicas, usando la definición de convolución. Sean $x, y \in \ell^{1}\left(\mathbb{N}_{0}\right)$, su convolución esta definida por

$$
\begin{aligned}
x * y= & \left(\sum_{k=0}^{n} x_{k} y_{n-k}\right)_{n \geq 0} \\
= & \left(x_{0} y_{0}, x_{0} y_{1}+x_{1} y_{0}, x_{0} y_{2}+x_{1} y_{1}+x_{2} y_{0},\right. \\
& \left.x_{0} y_{3}+x_{1} y_{2}+x_{2} y_{1}+x_{3} y_{0}, \ldots\right) .
\end{aligned}
$$

Ejemplo 4. Sea $\mathcal{B}$ el operador traslación a la izquierda sobre $\ell^{1}$. Entonces la función bilineal

$$
B(x, y)=x *(\mathcal{B} y)
$$

es bihipercíclica sobre $\ell^{1}$.

Esto sigue de la Proposición 1 y del hecho que

$$
\begin{aligned}
B\left(2 e_{0}, y\right) & =2 e_{0} *(\mathcal{B} y), \\
& =(2,0,0, \ldots) *\left(y_{1}, y_{2}, y_{3}, \ldots\right), \\
& =\left(2 y_{1}, 2 y_{2}+0 y_{1}, 2 y_{3}+0 y_{2}+0 y_{1}, 2 y_{4}+0 y_{3}+0 y_{2}+0 y_{1}, \ldots\right), \\
& =\left(2 y_{1}, 2 y_{2}, 2 y_{3}, 2 y_{4}, \ldots\right), \\
& =2 \mathcal{B} y,
\end{aligned}
$$


así $B\left(2 e_{0}, \cdot\right)=2 \mathcal{B}$ es hipercíclico, donde $e_{0}=(1,0,0, \ldots)$. De tal manera que, $B$ es bihipercíclica sobre $\ell^{1}$.

Asimismo, la simetría invariante sobre $B$ definida como

$$
B(x, y)=x *(\mathcal{B} y)+(\mathcal{B} x) * y
$$

es también bihipercíclica, ya que,

$$
\begin{aligned}
B\left(2 e_{0}, y\right)= & 2 e_{0} *(\mathcal{B} y)+\mathcal{B}(2,0,0, \ldots) * y \\
= & (2,0,0, \ldots) *\left(y_{1}, y_{2}, y_{3}, \ldots\right)+(0,0,0, \ldots) *\left(y_{0}, y_{1}, y_{2}, \ldots\right), \\
= & \left(2 y_{1}, 2 y_{2}+0 y_{1}, 2 y_{3}+0 y_{2}+0 y_{1}, 2 y_{4}+0 y_{3}+0 y_{2}+0 y_{1}, \ldots\right)+ \\
& \left(0 y_{0}, 0 y_{1}+0 y_{0}, 0 y_{2}+0 y_{1}+0 y_{0}, 0 y_{3}+0 y_{2}+0 y_{1}+0 y_{0}, \ldots\right), \\
= & \left(2 y_{1}, 2 y_{2}, 2 y_{3}, 2 y_{4}, \ldots\right)+(0,0,0, \ldots), \\
= & 2 \mathcal{B} y .
\end{aligned}
$$

\section{Bihiperciclicidad y n-upla hipercíclica}

Esta sección permite conectar el nuevo concepto de funciones bilineales bihipercíclicas con las $n$-uplas hipercíclicas. Una $n$-upla de operadores se define como $\mathbf{T}=\left(T_{1}, T_{2}, \ldots, T_{n}\right)$, donde $\mathbf{T} \in \mathcal{L}(X)^{n}$ conmuta, es decir, $T_{j} T_{k}=T_{k} T_{j}$ para $1 \leq j, k \leq n$, la cual es hipercíclica si existe un $x \in X$ tal que

$$
\operatorname{orb}(y, \mathbf{T})=\left\{T_{1}^{b_{1}} T_{2}^{b_{2}} \ldots T_{n}^{b_{n}}(x): b_{i} \in \mathbb{N}_{0}\right\}
$$

es densa en $X$.

Proposición 2. Sea $B$ una función bilineal tal que hay algún $x \in X$ para el cual los operadores $T_{1}=B(x, \cdot), T_{2}=B\left(T_{1} x, \cdot\right), \ldots, T_{n}=B\left(T_{n-1} x, \cdot\right)$ conmutan y forman un n-upla hipercíclica. Entonces $B$ es bihipercíclica. Más precisamente, si y es hipercíclico para esta $n$-upla, entonces $(x, y)$ es bihipercíclico para $B$.

Demostración. Como la $n$-upla es hipercíclica, existe un $y \in X$ tal que

$$
\operatorname{orb}(y, \mathbf{T})=\left\{T_{1}^{b_{1}} T_{2}^{b_{2}} \ldots T_{n}^{b_{n}}(y): b_{i} \in \mathbb{N}_{0}\right\}
$$

es densa en $X$. Los elementos de la órbita de $y$ bajo $\mathbf{T}$, son de la forma:

$$
\begin{aligned}
& T_{1} T_{2}(y)=T_{1}\left(B\left(T_{1} x, y\right)\right)=B\left(x, B\left(T_{1} x, y\right)\right)=B(x, B(B(x, x), y)) \\
& T_{2} T_{1}(y)=T_{2}(B(x, y))=B\left(T_{1} x, B(x, y)\right)=B(B(x, x), B(x, y))
\end{aligned}
$$


estos son también elementos de la órbita de $(x, y)$ bajo $B$, así, se tiene que

$$
\left\{T_{1}^{b_{1}} T_{2}^{b_{2}} \ldots T_{n}^{b_{n}}(y): b_{i} \in \mathbb{N}_{0}\right\} \subset \operatorname{orb}((x, y), B),
$$

por lo tanto $B$ es bihipercíclica.

Ejemplo 5. Sea $T_{1}, \ldots, T_{n}$ una $n$-upla hipercíclica; suponga que $x \in X$, tal que $x, T_{1} x, \ldots, T_{n-1} x$ son linealmente independientes, se tiene que el conjunto $\left\{x, T_{1} x, \ldots, T_{n-1} x\right\} \subset X$, entonces aplicando el Teorema de Hahn-Banach (Si $M$ es un subespacio de $X$ y $x_{0} \notin M$ entonces existe $x^{*} \in X^{*}$ tal que $x^{*}\left(x_{0}\right)=0$ y $x^{*}\left(x_{0}\right)=1$ si $\left.x_{0} \in M\right)$, se pueden seleccionar funcionales lineales $x_{k}^{*} \in X^{*}$, tal que $x_{k}^{*}\left(T_{l-1} x\right)=\delta_{k, l}$, con valores de $k, l=1,2, \ldots, n$, donde $T_{0}=I$ y $\delta_{k, l}=1$ cuando $k=l$, de lo contrario $\delta_{k, l}=0\left(\delta_{k, l}\right.$ llamado delta de Kronecker). Entonces

$$
B=\sum_{k=1}^{n} x_{k}^{*} \otimes T_{k}
$$

es bihipercíclica.

En forma general, sea $T_{1}, \ldots, T_{n}$ una $n$-upla hipercíclica, suponga que $x \in$ $X$, tal que $x, T_{1} x, \ldots, T_{n-1} x$ son linealmente independientes, aplicando la definición de $\otimes \mathrm{y}$ de los $x_{k}^{*}$ en (5), se tiene

$$
\begin{aligned}
B(x, y)= & \sum_{k=1}^{n} x_{k}^{*} \otimes T_{k}(x, y) \\
= & x_{1}^{*} \otimes T_{1}(x, y)+x_{2}^{*} \otimes T_{2}(x, y)+x_{3}^{*} \otimes T_{3}(x, y)+\ldots+ \\
& +x_{n}^{*} \otimes T_{n}(x, y) \\
= & x_{1}^{*}(x) T_{1}(y)+x_{2}^{*}(x) T_{2}(y)+x_{3}^{*}(x) T_{3}(y)+\ldots+x_{n}^{*}(x) T_{n}(y), \\
= & 1 T_{1}(y)+0 T_{2}(y)+0 T_{3}(y)+\ldots+0 T_{n}(y) \\
= & T_{1}(y) \\
B\left(T_{1} x, y\right)= & \sum_{k=1}^{n} x_{k}^{*} \otimes T_{k}\left(T_{1} x, y\right) \\
= & x_{1}^{*} \otimes T_{1}\left(T_{1} x, y\right)+x_{2}^{*} \otimes T_{2}\left(T_{1} x, y\right)+\ldots+x_{n}^{*} \otimes T_{n}\left(T_{1} x, y\right), \\
= & x_{1}^{*}\left(T_{1} x\right) T_{1}(y)+x_{2}^{*}\left(T_{1} x\right) T_{2}(y)+x_{3}^{*}\left(T_{1} x\right) T_{3}(y)+\ldots+ \\
& +x_{n}^{*}\left(T_{1} x\right) T_{n}(y), \\
= & 0 T_{1}(y)+1 T_{2}(y)+0 T_{3}(y)+\ldots+0 T_{n}(y), \\
= & T_{2}(y),
\end{aligned}
$$

Rev.Mate.Teor.Aplic. (ISSN print: 1409-2433; online: 2215-3373) Vol. 24(1): 29-44, January 2017 


$$
\begin{aligned}
B\left(T_{2} x, y\right)= & \sum_{k=1}^{n} x_{k}^{*} \otimes T_{k}\left(T_{2} x, y\right) \\
= & x_{1}^{*} \otimes T_{1}\left(T_{2} x, y\right)+x_{2}^{*} \otimes T_{2}\left(T_{2} x, y\right)+x_{3}^{*} \otimes T_{3}\left(T_{2} x, y\right)+\ldots+ \\
& +x_{n}^{*} \otimes T_{n}\left(T_{2} x, y\right) \\
= & x_{1}^{*}\left(T_{2} x\right) T_{1}(y)+x_{2}^{*}\left(T_{2} x\right) T_{2}(y)+x_{3}^{*}\left(T_{2} x\right) T_{3}(y)+\ldots+ \\
& +x_{n}^{*}\left(T_{2} x\right) T_{n}(y) \\
= & 0 T_{1}(y)+0 T_{2}(y)+1 T_{3}(y)+\ldots+0 T_{n}(y) \\
= & T_{3}(y)
\end{aligned}
$$

y de la misma manera se procede con $B\left(T_{3} x, y\right), B\left(T_{4} x, y\right), \ldots, B\left(T_{n-1} x, y\right)$. Por lo tanto, con $y$ hipercíclico para esta $n$-upla entonces $(x, y)$ es bihipercíclico para $B$, así $B$ es bihipercíclica.

En particular, se demostrará que $B(x, y)=x_{2} \mathcal{B} y+2 x_{1} y$ es una función bilineal bihipercíclica. Sea $\mathcal{B}: \ell^{2} \rightarrow \ell^{2}$ el operador traslación a la izquierda e $I$ el operador identidad, $T_{k}=(\mathcal{B}, 2 I)$ es un par hipercíclico, ver [5]. Ahora, sea $x=\left(x_{1}, x_{2}, x_{3}, \ldots\right)=(0,1,0,0, \ldots) \in \ell^{2}$, donde $x, T_{1}(x)$ son linealmente independientes; se define $x_{1}^{*}: \ell^{2} \rightarrow \mathbb{C}$ dado por $x_{1}^{*}(x)=x_{2}$ y $x_{2}^{*}: \ell^{2} \rightarrow \mathbb{C}$ como $x_{2}^{*}(x)=x_{1}$.

Así $x_{1}^{*}$ aplicado a $x$ y a $T_{1}(x)$,

$$
\begin{aligned}
& x_{1}^{*}\left(T_{0} x\right)=x_{1}^{*}(x)=x_{2}=1, \\
& x_{1}^{*}\left(T_{1} x\right)=x_{1}^{*}(\mathcal{B}(x))=x_{1}^{*}\left(x_{2}, x_{3}, x_{4}, \ldots\right)=x_{1}^{*}(1,0,0,0, \ldots)=0,
\end{aligned}
$$

y $x_{2}^{*}$ aplicado a $x$ y a $T_{1}(x)$

$$
\begin{aligned}
& x_{2}^{*}\left(T_{0} x\right)=x_{2}^{*}(x)=x_{1}=0, \\
& x_{2}^{*}\left(T_{1} x\right)=x_{2}^{*}(\mathcal{B}(x))=x_{2}^{*}(1,0,0,0, \ldots)=1,
\end{aligned}
$$

cumplen con las hipótesis del Ejemplo, entonces

$$
\begin{aligned}
B(x, y) & =\sum_{k=1}^{2} x_{k}^{*} \otimes T_{k}(x, y), \\
& =x_{1}^{*} \otimes T_{1}(x, y)+x_{2}^{*} \otimes T_{2}(x, y), \\
& =x_{1}^{*}(x) T_{1}(y)+x_{2}^{*}(x) T_{2}(y), \\
& =x_{2} \mathcal{B} y+2 x_{1} y,
\end{aligned}
$$

es bihipercíclica. Por lo tanto, con $y$ hipercíclico para este par de operadores entonces $(x, y)$ es bihipercíclico para $B$. 
Es importante mencionar, que aunque Feldman [5] demostró que existe una $(n+1)$-upla $\left(T_{1}, T_{2}, \ldots, T_{n}, T_{n+1}\right)$ de matrices diagonales hipercíclica sobre $\mathbb{C}^{n}$, este resultado, no puede ser aplicado para demostrar la existencia de funciones bilineales bihipercíclicas, ya que para un $x \in \mathbb{C}^{n}$, se tiene que $x, T_{1} x$, $T_{2} x, \ldots, T_{n} x$ son linealmente dependientes. Por otra parte, Shakarin [12] demostró que el minimal (valor más pequeño) de $N$, para que una $N$-upla de matrices diagonales sea hipercíclica sobre $\mathbb{C}^{n}$ es $n+1$.

\section{Referencias}

[1] Ansari, S. (1997) "Existence of hypercyclic operators on topological vector spaces”, J. Funct. Anal. 148(2): 384-390.

[2] Bayart, F.; Matheron, É. (2009) Dynamics of Linear Operator. Cambridge Tracts in Mathematics, Cambridge University Press.

[3] Bernal-González, L. (1999) "On hypercyclic operators on Banach spaces", Proc. Amer. Math. Soc. 127(4): 1003-1010.

[4] Costakis, G.; Hadjiloucas, D.; Manoussos, A. (2009) "Dynamics of tuples of matrices”, American Mathematical Society 137(3): 1025-1034.

[5] Feldman, N. (2008) "Hypercyclic tuples of operators and somewhere dense orbits", J. Math. Anal. Appl. 346(1): 82-98.

[6] Grosse-Erdmann, K.; Kim, S. (2013) "Bihypercyclic bilinear mappings", Journal of Mathematical Analysis and Applications 399(2): 701-708.

[7] Grosse-Erdmann, K.; Peris, A. (2011) Linear Chaos. Springer-Verlag, London-New York.

[8] Kitai, C. (1982) Invariant Closed Sets for Linear Operators. Ph.D. thesis, Universidad de Toronto, Canada.

[9] León, F.; Muller, V. (2004) "Rotations of hypercyclic and supercyclic operators", Integral Equations Operator Theory 50(1): 385-391.

[10] Rolewicz, S. (1969) “On orbits of elements”, Studia Math. 32(1): 17-22.

[11] Shapiro, J. (2001) "Notes on the dynamics of linear operators", en: http://www.mth.msu.edu/ shapiro/Pubvit/Downloads / LinDynamics $\backslash /$ lindynamics.pdf, consultada 11-May-2013, 8:00 a.m. 
[12] Shkarin, S. (2011) "Hypercyclic tuples of operators on $\mathbb{C}^{n}$ and $\mathbb{R}^{n \text { ", Linear }}$ and Multilinear Algebra 60(8): 885-896. 